\title{
A Fifth-Order Stokes Theory FOR STEADY WAVES
}

\author{
By John D. Fenton ${ }^{1}$
}

\begin{abstract}
An alternative Stokes theory for steady waves in water of constant depth is presented where the expansion parameter is the wave steepness itself. The first step in application requires the solution of one nonlinear equation, rather than two or three simultaneously as has been previously necessary. In addition to the usually specified design parameters of wave height, period and water depth, it is also necessary to specify the current or mass flux to apply any steady wave theory. The reason being that the waves almost always travel on some finite current and the apparent wave period is actually a Dopplershifted period. Most previous theories have ignored this, and their application has been indefinite, if not wrong, at first order. A numerical method for testing theoretical results is proposed, which shows that two existing theories are wrong at fifth order, while the present theory and that of Chappelear are correct. Comparisons with experiments and accurate numerical results show that the present theory is accurate for wavelengths shorter than ten times the water depth.
\end{abstract}

\section{INTRODUCTION}

The problem of a steadily-progressing periodic wave train is perhaps the simplest problem in the field of water waves, and yet because of the nonlinear nature of the governing equations, has been solved accurately only in recent years, during which several interesting phenomena have been discovered. For a recent survey of the problem and various solutions, reference can be made to Schwartz and Fenton (10). For practical problems, an application-oriented accurate method is that of Rienecker and Fenton (8), however this and all others which obtain accurate solutions even for high waves are based on numerical methods, and are not presented as explicit solutions. In problems where the waves are not very high or where great accuracy is not required, it is more reasonable to use an approximate explicit solution, such as cnoidal theory for shallow water or Stokes' theory for deeper water.

The essential features of Stokes' theory for periodic steady waves are: all variation in the direction of propagation is represented by Fourier series, and the coefficients in these series can be written as perturbation expansions in terms of a parameter which increases with wave height. Stokes used $a k$, the leading term in a Fourier series, in which $k=$ the wave number, $k=2 \pi / \lambda$; and $\lambda=$ the wavelength, and $a$ has no physical significance other than that of being a length scale which is equal to the amplitude of the wave at lowest order. The terms in the perturbation expansion can be found by satisfying boundary conditions on the free surface, and solving the resulting set of ordered equations.

The shortest way of proceeding to a solution is by an inverse method, in which the complex coordinate in the physical plane is obtained as a

\footnotetext{
${ }^{1}$ Sr. Lect., School of Mathematics, Univ. of New South Wales, Kensington, N.S.W., Australia 2033.

Note.-Discussion open until August 1, 1985. To extend the closing date one month, a written request must be filed with the ASCE Manager of Journals. The manuscript for this paper was submitted for review and possible publication on March 18, 1983. This paper is part of the Journal of Waterway, Port, Coastal and Ocean Engineering, Vol. 111, No. 2, March, 1985. CASCE, ISSN 0733-950X/ 85/0002-0216/\$01.00. Paper No. 19605.
} 
function of the complex velocity potential. In this case the boundaries of the problem are known initially, as the stream function is constant on the bottom and on the free surface. For arbitrary constant depth, Stokes obtained a solution to third order in $a k$. This was taken to fifth order by De (4). Subsequently, computer manipulation of the series was used by Schwartz (9) and Cokelet (2) to obtain numerical values of the coefficients for a given ratio of depth to wavelength to very high orders. Schwartz found that use of the leading coefficient, $a k$, as the expansion parameter had an important limitation. ak reaches a maximum before the highest wave is attained, with the result that convergence of the series is limited. This was partially overcome by using the actual crestto-trough wave height, $H$, in the expansion parameter, as $k H / 2$, but it was necessary to use convergence-enhancement procedures to obtain accurate results for steep waves.

For the solution of many practical problems, the inverse plane results are not readily applicable. De (4) reverted his series to give an expression for the complex potential, from which velocities can be obtained as a function of position. Similar fifth-order results were presented by Chappelear (1), who suggested that De's results were wrong at fifth order. For practical application, however, this approach has a limitation in that the theory is presented in terms of $a k$ and $k l$, in which $l=$ a depth scale given by the ratio of the volume flux under the stationary waves to the mean fluid velocity on a constant level. It is not, in general, equal to the depth, and is initially unknown. This causes difficulty in practical problems as neither $a$ nor $l$, and usually not $k$, are known a priori. They have to be obtained through the solution of three simultaneous nonlinear equations. It is difficult to obtain a solution to these equations in the limit of shallow water and, surprisingly, also in the deep water limit. An approximate procedure for the latter case has been given by Dailey (3).

Skjelbreia and Hendrickson (11) obtained an alternative fifth-order Stokes theory, proceeding via the physical plane. They obtained a solution in terms of $a k$ and $k d$, in which $d=$ the mean fluid depth. Their theory is slightly simpler to apply than those obtained via the inverse plane, for only two nonlinear simultaneous equations have to be solved for $a$ and $k$. However, obtaining a solution is still often difficult. It will be shown in the following that the Skjelbreia and Hendrickson theory is, in fact, wrong at fifth order.

All of the theories described previously have made the implicit assumption that the waves travel at Stokes' first definition of wave speed. As a result, in the frame of interest, they travel such that the time meanfluid velocity at all points within the fluid is zero. This need not be the case, of course. Indeed, it is much more likely that the waves are travelling on some small but finite current, whether it be positive or negative, and the measured period is a Doppler-shifted period. The theories do not determine the wave speed-it is determined by external physical factors, such as the current, or, in other situations, by the mass or volume flux underneath the waves. Tsuchiya and Yamaguchi (12) have developed a fourth-order theory in which it is assumed that the waves travel at Stokes' second definition of wave speed, such that the mass flux is zero. While this is the case for experiments in a closed tank, in 
general it need not be true either. In the general case, to apply any steady wave theory it is usually necessary to know either the wave speed or the current or the mass flux. This has been pointed out by Rienecker and Fenton (8), and allowed for in their numerical method.

In this paper a fifth-order Stokes theory for steady waves is presented in which an attempt is made to correct and to obviate some of the disadvantages of previous theories. Instead of an unknown Fourier coefficient $a k$ being used as expansion parameter, the dimensionless wave height (wave steepness) is used in the form $k H / 2$. The expressions for the individual coefficients are functions only of the dimensionless depth, $k d$, so that the only unknown, ab initio, is the wave number $k$, which can be found by the numerical solution of a single nonlinear equation. Two alternative possibilities for this equation are given, one in terms of the mean current at a point, the other in terms of the mean mass (volume) flux under the waves. If neither of these quantities is known, nor any other factor which determines the wave speed, then there is little point in using the fifth-order theory or any other high-order theory. The results would be mathematically inconsistent at first order, and inclusion of higher order terms simply a waste of time.

The present solution and two previous fifth-order solutions are checked numerically, and it is shown that both De's and Skjelbreia and Hendrickson's theories are wrong at fifth order. Subsequently, application of the theory to practical problems is examined, showing when and how the present theory can be used. Finally, results from the present theory are compared with some experimental data, and with accurate results from numerical solutions.

\section{Steady Wave Equations and Their Solution}

The problem considered is that of periodic waves propagating without change of form over a layer of fluid on a horizontal impermeable bed. The origin is on the bed, the horizontal coordinate is $x$ and the vertical coordinate is $y$. This reference frame moves with the same velocity as the waves so that in this frame all motion is steady. The problem can be uniquely solved in this frame. Not until any other frame of reference

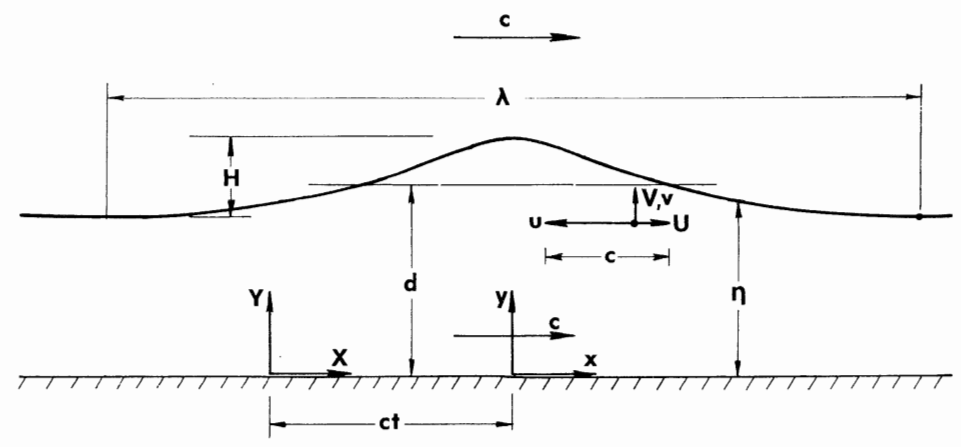

FIG. 1.-One Wave of Steady Periodic Wave Train, Showing Important Physical Quantities 
is considered does the wave speed have to be known. Fig. 1 shows the physical situation and important dimensions.

If the fluid is incompressible, a stream function, $\psi$, exists such that velocity components $(u, v)$ are given by $u=\partial \psi / \partial y, v=-\partial \psi / \partial x$, and, if the motion is irrotational, $\psi$ satisfies Laplace's equation throughout the fluid. Thus

$\frac{\partial^{2} \psi}{\partial x^{2}}+\frac{\partial^{2} \psi}{\partial y^{2}}=0$

The boundary conditions to be satisfied are

$\psi(x, 0)=0$

on the bottom $y=0$; on the free surface $y=\eta(x)$ the kinematic boundary condition is

$\psi[x, \eta(x)]=-Q$

in which $Q=$ a positive constant denoting the total volume rate of flow underneath the stationary wave per unit length normal to the $(x, y)$ plane; and the condition requiring pressure on the free surface to be constant, combined with Bernoulli's equation, gives

$\frac{1}{2}\left[\left(\frac{\partial \psi}{\partial x}\right)^{2}+\left(\frac{\partial \psi}{\partial y}\right)^{2}\right]+g \eta=R$

on the free surface $y=\eta(x)$, in which $g=$ the gravitational acceleration; and $R=$ a positive constant.

Three physical dimensions uniquely define a particular problem: the mean water depth, $d$, the wave height, $H$, and the wavelength, $\lambda$. A solution will be obtained here in terms of these quantities, although as will be examined subsequently, the wavelength is often initially unknown. It is more convenient to use the wave number $k=2 \pi / \lambda$ in the analysis, and to introduce the dimensionless wave amplitude $\epsilon=k H / 2$, often known as the wave steepness.

The following expansion for $\psi$ is assumed, as suggested by previous Stokes wave theories:

$\frac{k \psi}{\bar{u}}=-k y+\sum_{i=1}^{\infty} \sum_{j=1}^{i} f_{i j} \epsilon^{i} \sinh j k y \cos j k x$.

in which $\bar{u}=$ the mean fluid speed for any constant value of $y$. It is simpler to write it on the left side so that the leading term in the expansion is simply $-k y$, which greatly facilitates subsequent calculations. The coefficients $f_{i j}$ are dimensionless.

Eq. 5 satisfies the field Eq. 1 and the boundary condition on the bottom, Eq. 2. Substituting the expansion for $\psi$ into the surface boundary conditions Eqs. 3 and 4 gives

$\frac{k Q}{\bar{u}}-k \eta(x)+\sum_{i=1}^{\infty} \sum_{j=1}^{i} f_{i j} \epsilon^{i} \sinh j k \eta(x) \cos j k x=0$ 


$$
\begin{aligned}
& \text { and }\left[-1+\sum_{i=1}^{\infty} \sum_{j=1}^{i} j f_{i j} \epsilon^{i} \cosh j k \eta(x) \cos j k x\right]^{2} \\
& +\left[\sum_{i=1}^{\infty} \sum_{j=1}^{i} j f_{i j} \epsilon^{i} \sinh j k \eta(x) \sin j k x\right]^{2}+\frac{2 g}{\bar{u}^{2}} \eta(x)-\frac{2 R}{\bar{u}^{2}}=0 \ldots \ldots \ldots \ldots
\end{aligned}
$$

Now, perturbation expansions in terms of $\epsilon$ can be assumed for the quantities in these equations, in which the undisturbed state is a uniform flow (in the negative $x$ direction) of depth, $d$ and speed, $C_{o}(g / k)^{1 / 2}$ :

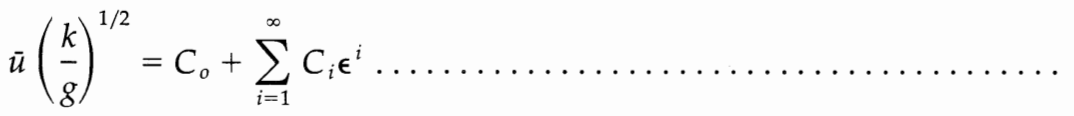

$$
\begin{aligned}
& Q\left(\frac{k^{3}}{g}\right)^{1 / 2}=\bar{u} d\left(\frac{k^{3}}{g}\right)^{1 / 2}+\sum_{i=1}^{\infty} D_{i} \epsilon^{i} \\
& \frac{R k}{g}=\frac{1}{2} C_{o}^{2}+k d+\sum_{i=1}^{\infty} E_{i} \epsilon^{i} \\
& \text { and } k \eta(x)=k d+\sum_{i=1}^{\infty} \sum_{j=1}^{i} b_{i j} \epsilon^{i} \cos ^{j} k x \text {. }
\end{aligned}
$$

The coefficients in these equations $\left(b_{i j}, C_{o}, C_{i}, D_{i}\right.$, and $\left.E_{i}\right)$ are all dimensionless. Eq. 11 is presented as a power series with terms like $\cos ^{j} k x$, rather than as a Fourier series with terms like $\cos j k x . \eta(x)$ appears in the argument of hyperbolic functions in Eqs. 6 and 7, and will be expanded as a power series because it is easier to do if the argument is a power series. As this series in Eq. 11 must be such that the mean value of $\eta(x)$ is $d$, and the difference between the crest and trough elevations is $H$, certain relations exist between coefficients in the power series.

If the expansions Eqs. 8-11 are substituted into Eqs. 6 and 7, then all the hyperbolic functions can be expanded (as power series of power series) and all the necessary series multiplications performed. As the boundary conditions must be satisfied for all values of $\epsilon$ and all values of $x$, a hierarchy of separate equations is obtained, each being the coefficient of a term in a double power series in $\epsilon$ and $\cos k x$. The number of terms in these equations increases rapidly with order. For example, those equations obtained from the Bernoulli equation contain 2 terms at order $\epsilon$, and successively $10,14,44$ and finally 83 at order $\epsilon^{5}$, after which calculations were terminated. These operations and subsequent solution of the equations took the writer about a week of intense effort (each time, until he got it right!) including a number of numerical checks at each stage. After subsequent multiplications and conversion to Fourier series, the following solution was obtained.

\section{Presentation of Solution}

In this section, the solution will be presented as a number of power 
series expansions, truncated after fifth order. The coefficients in these expansions, which are dimensionless, are given in Table 1 . Whereas it is simpler to use the stream function to obtain a solution, it seems rather more direct to present results in terms of the velocity potential $\phi(x, y)$, in which $u=\partial \phi / \partial x=\partial \psi / \partial y$, and $v=\partial \phi / \partial y=-\partial \psi / \partial x$. The solution can be presented entirely in terms of the dimensionless wave height, $\epsilon$ $=k H / 2$ and dimensionless water depth, $k d$, in which $k=$ the wavenumber, $k=2 \pi / \lambda$. The procedure for determining $k$ is given later in this paper for several different cases commonly encountered in practice. In some situations, notably when just wave height, period, and water depth are specified, it will be shown that it is unreasonable to use any theory higher than first order. It should be noted that in most practical applications it is desirable to know velocities, pressures and elevations in a frame through which the waves are moving. Expressions for these are given in the section headed "Application of Theory," where the procedure for determining $k$ is also given.

The solution to the steady wave problem is as follows:

$\phi(x, y)=-\bar{u} x+C_{o}\left(\frac{g}{k^{3}}\right)^{1 / 2} \sum_{i=1}^{5} \epsilon^{i} \sum_{j=1}^{i} A_{i j} \cosh j k y \sin j k x+0\left(\epsilon^{6}\right) \ldots \ldots$

in which the mean horizontal fluid speed, $\bar{u}$, is given by

$\bar{u}\left(\frac{k}{g}\right)^{1 / 2}=C_{o}+\epsilon^{2} C_{2}+\epsilon^{4} C_{4}+0\left(\epsilon^{6}\right) \ldots \ldots \ldots \ldots \ldots \ldots \ldots \ldots \ldots \ldots \ldots \ldots \ldots \ldots \ldots \ldots \ldots$

The Landau order symbol $O\left(\epsilon^{6}\right)$ means that neglected terms are of the order of $\epsilon^{6}$. It should be pointed out that $\bar{u}$ is the quantity usually referred to as the "wave speed" in presentations of wave theory, which is Stokes' first definition of wave speed. It is numerically equal to the wave speed only relative to a frame in which the mean current is zero. This will be further examined in the following.

The expression for the free surface profile is

$$
\begin{aligned}
& k \eta(x)=k d+\epsilon \cos k x+\epsilon^{2} B_{22} \cos 2 k x+\epsilon^{3} B_{31}(\cos k x-\cos 3 k x) \\
& +\epsilon^{4}\left(B_{42} \cos 2 k x+B_{44} \cos 4 k x\right)+\epsilon^{5}\left(-\left(B_{53}+B_{55}\right) \cos k x\right. \\
& \left.+B_{53} \cos 3 k x+B_{55} \cos 5 k x\right)+0\left(\epsilon^{6}\right) \ldots \ldots \ldots \ldots \ldots \ldots \ldots \ldots \ldots \ldots \ldots \ldots \ldots \ldots \ldots \ldots
\end{aligned}
$$

while the expansion for the volume flux under the wave is

$$
\begin{aligned}
& Q\left(\frac{k^{3}}{g}\right)^{1 / 2}=C_{o} k d+\epsilon^{2}\left(C_{2} k d+D_{2}\right)+\epsilon^{4}\left(C_{4} k d+D_{4}\right) \\
& +O\left(\epsilon^{6}\right)=\tilde{u} d\left(\frac{k^{3}}{g}\right)^{1 / 2}+\epsilon^{2} D_{2}+\epsilon^{4} D_{4}+0\left(\epsilon^{6}\right) \ldots \ldots \ldots \ldots \ldots \ldots \ldots
\end{aligned}
$$

and that for the Bernoulli constant is

$$
\frac{R k}{g}=\frac{1}{2} C_{o}^{2}+k d+\epsilon^{2} E_{2}+\epsilon^{4} E_{4}+0\left(\epsilon^{6}\right)
$$




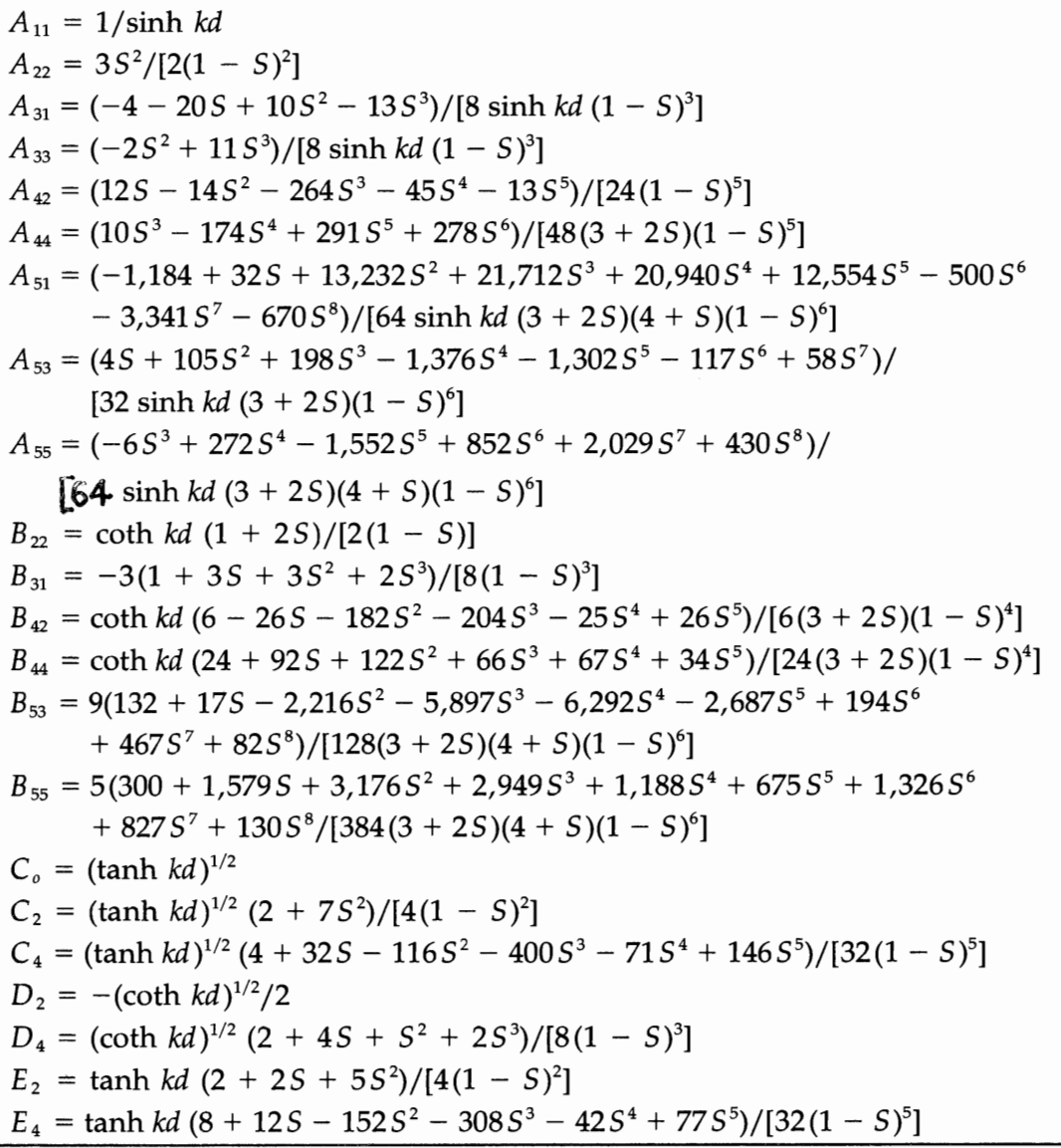

an terms of hyperbolic functions of $k d$, including $S=\operatorname{sech} 2 k d$.

The dimensionless coefficients with numerical subscripts in these equations are functions only of $k d$. The formulas for the individual coefficients can be presented most conveniently in terms of the hyperbolic function $S=$ sech $2 k d$, and other hyperbolic functions, and are given in Table 1.

There are two important limits for $k d$. The asymptotic behavior of the coefficients for the deep-water limit, $k d \rightarrow \infty$, is shown in Col. 2 of Table 2 , while in Col. 3 the shallow water limiting behavior of the coefficients as $k d \rightarrow 0$ are shown. Finally, in Col. 4 the numerical values of each for a particular value of $d / \lambda=0.12, k d=0.753982 \ldots$ are given. This is the value used by Skjelbreia and Hendrickson in the worked example they presented. The writer found this very helpful in numerical checking of their work and of the present work, and which may provide a check if others write programs from the formulas in Table 1.

An examination of results for the two depth limits is now given: 


\begin{tabular}{|c|c|c|c|}
\hline $\begin{array}{l}\text { Coefficient } \\
\text { (1) }\end{array}$ & $\begin{array}{l}\text { Deep water, value in } \\
\text { limit as } k d \rightarrow \infty \\
\text { (2) }\end{array}$ & $\begin{array}{c}\text { Shallow water, } \\
\text { behavior in limit } \\
\text { as } k d \rightarrow 0 \\
\text { (3) }\end{array}$ & $\begin{array}{c}\text { Value for } \\
k d=0.753982 \\
(4)\end{array}$ \\
\hline$A_{11}$ & $2 e^{-k d}$ & $\sim(k d)^{-1 \mathrm{~b}}$ & 1.208490 \\
\hline$A_{22}$ & $6 e^{-4 k d}$ & -4 & 0.799840 \\
\hline$A_{31}$ & $-e^{-k d}$ & -7 & -9.105340 \\
\hline$A_{33}$ & $-2 e^{-5 k d}$ & -7 & 0.368275 \\
\hline$A_{42}$ & $e^{-2 k d}$ & -10 & -12.196150 \\
\hline$A_{44}$ & $5 e^{-6 k d} / 9$ & -10 & 0.058723 \\
\hline$A_{51}$ & $-37 e^{-k d} / 12$ & -13 & 108.467921 \\
\hline$A_{53}$ & $e^{-3 k d} / 6$ & -13 & -6.941756 \\
\hline$A_{55}$ & $-e^{-7 k d} / 8$ & -13 & -0.074979 \\
\hline$B_{22}$ & $1 / 2$ & -3 & 2.502414 \\
\hline$B_{31}$ & $-3 / 8$ & -6 & -5.731666 \\
\hline$B_{42}$ & $1 / 3$ & -9 & -32.407508 \\
\hline$B_{44}$ & $1 / 3$ & -9 & 14.033758 \\
\hline$B_{53}$ & $99 / 128$ & -12 & -103.445042 \\
\hline$B_{55}$ & $125 / 384$ & -12 & 37.200027 \\
\hline$C_{0}$ & 1 & $1 / 2$ & 0.798448 \\
\hline $\mathrm{C}_{2}$ & $1 / 2$ & $-7 / 2$ & 1.940215 \\
\hline$C_{4}$ & $1 / 8$ & $-19 / 2$ & -12.970403 \\
\hline$D_{2}$ & $-1 / 2$ & $-1 / 2$ & -0.626215 \\
\hline$D_{4}$ & $1 / 4$ & $-13 / 2$ & 3.257104 \\
\hline$E_{2}$ & $1 / 2$ & -3 & 1.781926 \\
\hline$E_{4}$ & $1 / 4$ & -9 & -11.573657 \\
\hline
\end{tabular}

${ }^{a}$ The limiting value in the deep water limit is given, but for shallow water the behavior only is given.

${ }^{\mathrm{b}}$ After the first entry, only the exponent of $k d$ is given.

Deep Water $k d \rightarrow \infty$.-The results in Col. 2 of Table 2 are easily obtained from the expressions in Table 1 , because as $k d \rightarrow \infty, S=$ sech $2 k d$ $\approx e^{-2 k d} \rightarrow 0$. It is this limit in which the Stokes theory is most accurate. Incorporating the limiting forms into Eq. 12, with the limiting form of cosh $j k y$ and introducing the coordinate $y_{*}=y-d$, the elevation above the mean surface level, gives

$$
\begin{aligned}
& \left(\frac{k^{3}}{g}\right)^{1 / 2} \phi(x, y)=-k x\left(1+\frac{1}{2} \epsilon^{2}+\frac{1}{8} \epsilon^{4}\right)+\epsilon e^{k y *} \sin k x-\frac{1}{2} \epsilon^{3} e^{k y *} \sin k x \\
& +\frac{1}{2} \epsilon^{4} e^{2 k y_{*}} \sin 2 k x+\epsilon^{5}\left(\frac{-37}{24} e^{k y_{*}} \sin k x+\frac{1}{12} e^{3 k y_{*}} \sin 3 k x\right)+O\left(\epsilon^{6}\right)
\end{aligned}
$$

It is interesting that in this deep water limit, the higher frequency terms for a given order disappear, and this fifth order result contains only the first three harmonics. By considering the deep water limit for Eq. 14, the expression for $\eta_{*}(x)$, the height of the free surface above the mean in deep water can be obtained: 


$$
\begin{aligned}
& k \eta_{*}(x)=\epsilon \cos k x+\frac{1}{2} \epsilon^{2} \cos 2 k x+\frac{3}{8} \epsilon^{3}(\cos 3 k x-\cos k x) \\
& +\frac{1}{3} \epsilon^{4}(\cos 2 k x+\cos 4 k x)+\frac{1}{384} \epsilon^{5}(-422 \cos k x+297 \cos 3 k x \\
& +125 \cos 5 k x)+0\left(\epsilon^{6}\right) \ldots \ldots \ldots \ldots \ldots \ldots \ldots \ldots \ldots \ldots \ldots \ldots \ldots \ldots \ldots \ldots \ldots \ldots \ldots
\end{aligned}
$$

while the expression for the mean fluid speed becomes

$$
\bar{u}\left(\frac{k}{g}\right)^{1 / 2}=1+\frac{1}{2} \epsilon^{2}+\frac{1}{8} \epsilon^{4}+0\left(\epsilon^{6}\right) .
$$

Shallow Water $k d \rightarrow 0$.- In this case $S \approx 1-2(k d)^{2}$. Results are given in Col. 3 of Table 2. It is clear that for each higher order term the coefficients vary like an extra power of $(k d)^{-3}$. Thus, the effective expansion parameter is $\epsilon /(k d)^{3}$ in the shallow water limit. As the limit considered is $k d \rightarrow 0$, this obviously is an inappropriate limit for the application of Stokes-type theories. They should only be applied if the magnitudes of both $\epsilon$ and $\epsilon /(k d)^{3}$ are small, and the magnitude of the latter parameter should be monitored if Stokes' theory is to be used in shallow water. This was first pointed out by Ursell (13), and the ratio, or one proportional to it, $H \lambda^{2} / d^{3}$, is known as the Ursell number. If this number is large, such that the higher-order contributions in the series are not small, then the theory should not be used. The region of validity of the theory is indicated by the results shown in Fig. 2. If the waves are long, but

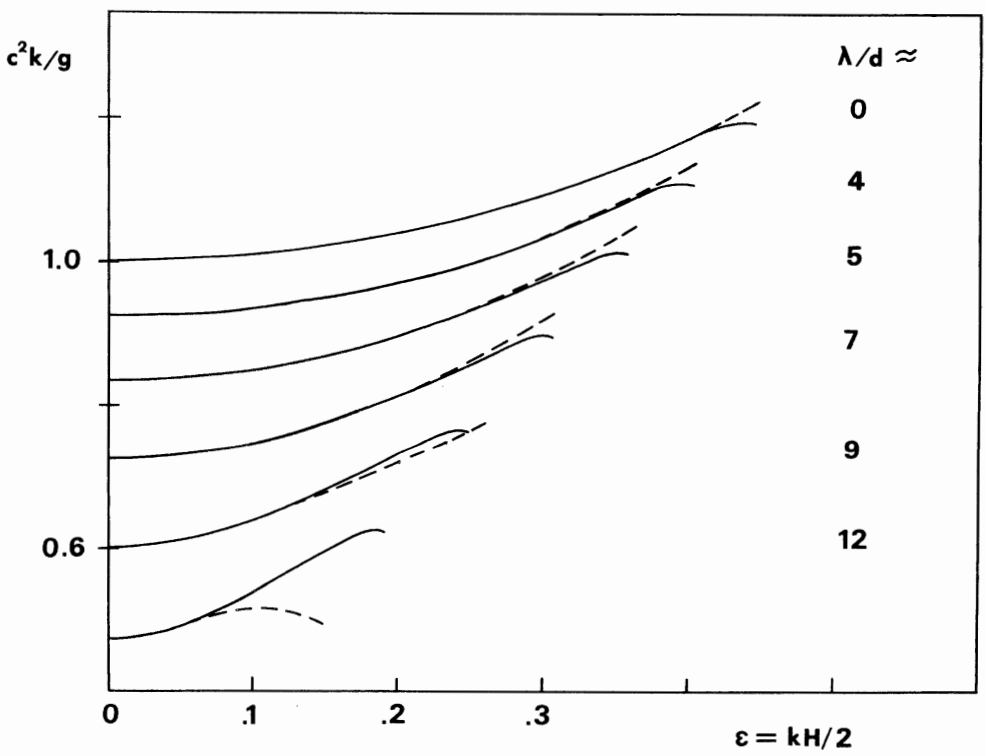

FIG. 2.-Comparison of Present Theory (Dashed Line) and High-Order Results of Cokelet (Solid Line) for Square of Stokes' First Definition of Wave Speed, as Function of Wave Height and Length 
not higher than about $40 \%$ of the water depth, then cnoidal theory can be used (5).

\section{Numerical Check of Fifth-Order Stokes Wave Theories}

A convenient numerical check can be made of the various fifth-order Stokes wave theories of Chappelear (1), De (4), Skjelbreia and Hendrickson (11) and the present work, by using a variant of the procedure known as (Richardson) extrapolation to the limit. The method should be capable of wider application in engineering and physics as a numerical means of checking theoretical results. To test each steady wave theory, the procedure is as follows. For any value of $k d$ (or $k l=k Q / \bar{u}$ in the case of Chappelear and $\mathrm{De}$ ), all the values of the coefficients in each solution are calculated, and for a certain small value of the expansion parameter, the residual errors on the free surface in each of the kinematic and Bernoulli surface boundary conditions are calculated at a number of points. In each case the whole procedure is followed separately. Taking a discrete Fourier transform of these errors, the amplitude of the individual Fourier components of the residual errors can be calculated. This whole process can be repeated for a different small value of the expansion parameter. It is then assumed that the amplitude of the $j$ th Fourier component of the error, $e_{j}(\epsilon)$, is given by

$e_{j}(\epsilon)=\alpha(j) \epsilon^{n(j)}+O\left(\epsilon^{n+1}\right)$

in which $\alpha$ and $n$ are independent of $\epsilon$. A numerical value of $n$, the order of the error terms of each harmonic in each free surface equation, can be calculated from the ratio of the errors computed for the two values of $\epsilon$, by the expression

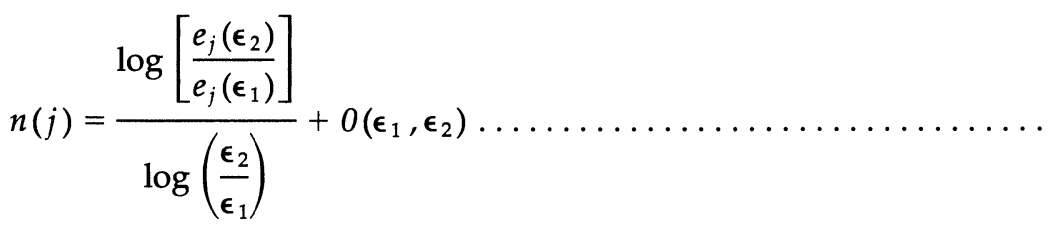

in which $\epsilon_{1}=$ the first value of $\epsilon$ used; and $\epsilon_{2}=$ the second. This was done using $\epsilon_{1}=0.01$ and $\epsilon_{2}=0.02$ for the theories of Chappelear, Skjelbreia and Hendrickson, and the present work, and the results are given in Table 3.

This is a demanding test, which checks the values of every term in every equation, and any error in the calculations would produce a lower value of $n$. The results are strong numerical evidence that Chappelear's theory is correct at fifth order, and so is the theory presented in this work, but that the theory of Skjelbreia and Hendrickson is wrong at fifth order in the dynamic boundary condition. It can also be noted, as Chappelear observed differences between his and De's results, that the latter's theory is also wrong at fifth order.

Skjelbreia and Hendrickson's theory (11) was examined by recasting the present theory to use their expansion parameter, which is the coefficient of $\cos k x$ in the series for the free surface profile. All the coefficients given by Skjelbreia and Hendrickson were obtained, with the sole 
TABLE 3.-Order of Errors in Kinematic and Dynamic Free Surface Boundary Conditions (Eqs. 3 and 4$)^{\mathrm{a}}$

\begin{tabular}{|c|c|c|c|c|c|c|}
\hline \multirow{2}{*}{$\begin{array}{c}\text { Fourier } \\
\text { component, } \\
j \\
\text { (1) }\end{array}$} & \multicolumn{2}{|c|}{ Chappelear } & \multicolumn{2}{|c|}{$\begin{array}{l}\text { Skjelbreia and } \\
\text { Hendrickson }\end{array}$} & \multicolumn{2}{|c|}{ Present Theory } \\
\hline & \multicolumn{2}{|l|}{ (and } & Kinem & ynamic & Kinematic & ynamic \\
\hline 0 & - & - & - & - & 6.0 & 6.0 \\
\hline 1 & 7.0 & 7.0 & 7.0 & $5.0^{\mathrm{b}}$ & 7.0 & 7.0 \\
\hline 2 & 6.0 & 6.0 & 6.0 & 6.0 & 6.0 & 6.0 \\
\hline 3 & 7.0 & 7.0 & 7.0 & 7.0 & 7.0 & 7.0 \\
\hline 4 & 6.0 & 6.0 & 6.0 & 6.0 & 6.0 & 6.0 \\
\hline 5 & 7.0 & 7.0 & 7.0 & 7.0 & 7.0 & 7.0 \\
\hline 6 & 6.0 & 6.0 & 6.0 & 6.0 & 6.0 & 6.0 \\
\hline 7 & 7.0 & 7.0 & 7.0 & 7.0 & 7.0 & 7.0 \\
\hline
\end{tabular}

${ }^{\text {a }}$ The numbers shown are $n(j)$, where the error has been assumed to be proportional to $\epsilon^{n(j)}$.

${ }^{b}$ Indicates error at fifth order in the Skjelbreia and Hendrickson theory.

exception of an incorrect sign in their expression for " $C$," the fifth-order term in the series for "wave speed." In that expression, a coefficient of $+2,592$ should in fact be $-2,592$. This has been pointed out by Nishimura, et al. (7). As " $C$ " is used throughout in Skjelbreia and Hendrickson's theory, it can be concluded that all applications of that theory which have not incorporated the sign change have been wrong at fifth order. This is in addition to the assertion in the following section that many applications of steady wave theories have been inconsistent at first order.

\section{Application OF TheORY}

Calculation of Wave Number $k$.-The solution for steady waves has been presented in the foregoing in terms of the two dimensionless quantities $\epsilon=k H / 2=\pi H / \lambda$, and $k d=2 \pi d / \lambda$. If the wave height, $H$, wavelength, $\lambda$, and water depth, $d$, are known, both $\epsilon$ and $k d$ can be calculated and the solution obtained. Often, however, the wave period, $\tau$, rather than the wave length is known initially, and often it is desirable to calculate the unsteady water velocities in a frame through which the waves are moving. In either of these situations it is necessary to know the wave speed, $c$, by measurement or from the following theory. It should be remembered that the theory itself can only predict the speed at which the waves travel relative to a frame in which there is zero current. The wave speed relative to a physical frame of interest, such as the sea bed, is determined by physical quantities external to the present solution, such as the current or the mass flux. The apparent period in that frame is a Doppler-shifted period. If neither the current nor the mass flux nor the wave speed is known, then it is theoretically inconsistent and practically a waste of time to use any theories other than the lowest order. The procedure in the various cases will be described in the following: 
1. $H, d$ and $\lambda$ Are Known.-The theory can be directly applied; however, if unsteady fluid velocities are to be calculated, a knowledge of the wave speed, $c$, is essential.

2. $H, d, \tau$ and $c$ Are Known.-In this case the wavelength $\lambda=c \tau$ and the procedure of case 1 can be followed.

3. H, $d, \tau$ and Mean Uniform Current Are Known.-This is the case expected to be most commonly encountered in practice. If the mean uniform current under the waves is known, the solution can be found. If the current is not known, reference should be made to case 5, which follows.

In a frame moving with the waves, the mean fluid speed is $\bar{u}$ and is in the negative $x$ direction, so that if the waves travel at speed $c$ in the positive $x$-direction, the Eulerian time mean fluid velocity at any point in the water, $c_{E}$, is given by

$c_{E}=c-\bar{u}$

This would be the mean current measured by a stationary velocity meter in the fluid under the waves. Now, $c=\lambda / \tau=2 \pi / k \tau$, and $\bar{u}$ is given by Eq. 13 as a function of $k H / 2$ and $k d$, which gives the transcendentallynonlinear equation to be solved for $k$ :

$\left(\frac{k}{g}\right)^{1 / 2} c_{E}-\frac{2 \pi}{\tau(g k)^{1 / 2}}+C_{o}(k d)+\left(\frac{k H}{2}\right)^{2} C_{2}(k d)$
$+\left(\frac{k H}{2}\right)^{4} C_{4}(k d)=0 \ldots \ldots \ldots \ldots \ldots \ldots \ldots \ldots \ldots \ldots \ldots \ldots \ldots \ldots \ldots \ldots \ldots \ldots \ldots \ldots \ldots \ldots \ldots \ldots \ldots$

in which the neglected terms of order $(\mathrm{kH} / 2)^{6}$ have not been shown. The functional dependence of $C_{0}, C_{2}$ and $C_{4}$ on $k d$ is indicated in this equation. The explicit details of this dependence are given in Table 1. For deep water, Col. 2 of Table 2 shows the numerical values of these coefficients, which are independent of $k d$ in the limit. The wave number, $k$, is the only unknown in this equation, and it can be determined by any of the usual iterative methods: trial and error, bisection, regula falsi, or by the secant method. The secant method is a convenient approximation to Newton's method where, as is the case here, the functional dependence on the unknown is sufficiently complicated so as to make differentiation rather daunting. A first approximation to be used in all solutions of Eq. 23 might be the linear deep-water result for small $c_{E}$, obtained from Eq. 23:

$k \approx \frac{4 \pi^{2}}{\tau^{2} g}\left(1-\frac{4 \pi c_{E}}{g \tau}\right)$.

Equations similar to Eq. 23 have been presented by Chappelear (1) and by Skjelbreia and Hendrickson (11) as part of a system of nonlinear equations ( 3 and 2 , respectively) to be solved to give the magnitudes of the expansion parameter and the wave number. In the present work the use of the actual wave height, $H$, in the expansion parameter and the actual depth, $d$, as the depth scale, means that only one equation has to be solved, a rather simpler task. 
A more important feature of Eq. 23 is the incorporation of the fact that waves may travel at any speed, and that the theory does not determine the actual wave speed. The mean current (the Eulerian mean horizontal fluid velocity) is a parameter to be specified, rather than assuming it to be identically zero as has been done by most wave theories, including the Stokes wave theories of Chappelear (1) and Skjelbreia and Hendrickson (11) and cnoidal wave theories, up to and including that of Fenton (5).

Once $k$ has been found, the theory can be applied, and the wave speed found from the equation $c=\bar{u}+c_{E}$.

4. $H, d, \tau$, and Mass or Volume Flux Known.-In some situations, notably in laboratory experiments, the mass flux under the waves is known or can be assumed, and this can be used to determine the solution. In a frame moving with the waves the volume flow rate underneath the waves per unit span is $Q$ (in the negative $x$-direction). Thus, the mean velocity with which the fluid is transported is $-Q / d$. In a frame through which the waves travel with velocity $c$, the mean fluid transport velocity in the direction of propagation of the waves (the "mass transport velocity" or "Stokes drift velocity") is $c_{S}$, such that

$c_{s}=c-\frac{Q}{d}$

Substituting this and $c=2 \pi / k \tau$ into Eq. 15 gives

$\left(\frac{k}{g}\right)^{1 / 2} c_{s}-\frac{2 \pi}{\tau(g k)^{1 / 2}}+C_{o}(k d)+\left(\frac{k H}{2}\right)^{2}\left[C_{2}(k d)+\frac{D_{2}(k d)}{k d}\right]$
$+\left(\frac{k H}{2}\right)^{4}\left[C_{4}(k d)+\frac{D_{4}(k d)}{k d}\right]=0 \ldots \ldots \ldots \ldots \ldots \ldots \ldots \ldots \ldots \ldots \ldots \ldots \ldots \ldots \ldots \ldots \ldots$

where the explicit functional dependence on $k d$ of $C_{0}, C_{2}, C_{4}, D_{2}$ and $D_{4}$ is given in Table 1 . The presence of neglected terms of sixth order has not been indicated. The wave number $k$ is the only unknown in this equation, and can be determined by the numerical methods previously mentioned in case 3. As a first approximation, Eq. 24 can be used, but where $c_{S}$ is used instead of $c_{E}$, the two are identical to second order.

Once $k$ has been found, the theory can be applied, and the wave speed found from the expression $c=Q / d+c_{S}$.

One common application of this mass flux condition rather than the mean current criterion of case 3 is to laboratory experiments in a tank with closed ends, in which $c_{S}=0$. In many previous comparisons between theory and experiment this has not been used, and the incorrect condition $c_{E}=0$ has been applied. It should be noted that Tsuchiya and Yamaguchi (12) have presented both Stokes and cnoidal wave theories for the condition $c_{S}=0$, but did not consider the general case, where $c_{S}$ may have a finite specified value.

5. $H, d$, and $\tau$ Only Are Known.-This has often been the situation in practice. If neither the wave speed, $c$, is known nor the mean current, $c_{E}$, nor the mass flux velocity, $c_{S}$, nor any other quantity which determines the wave speed, then there is no mathematically-rational basis for applying any wave theory. Neither Eq. 23 nor 26 can be solved for the 
wave number $k$. In most geophysical applications, however, both the mean current and the mass flux velocity are small compared with typical wave speeds. So as a sensible but mathematically-irrational approximation, a value of $c_{E}=0$ might be assumed, giving Stokes' first definition of wave speed, or a value of $c_{S}=0$ might be taken, giving his second definition. As the accuracy of this assumption is unknown in any particular situation, there would seem to be no justification for including any of the higher order terms in Eqs. 23 or 26, in which case they reduce to the same first-order approximation. Thus, there is no justification for any theory other than first-order theory to be used subsequently in this case.

Application of Theory to Propagating Waves.-To use the equations presented in this section it is necessary to have calculated $\epsilon$ and $k d$ from the previous theory, and to determine $c$ as described in each case. It is, of course, not necessary to use the full fifth-order theory. Depending on the particular application, the theory can be used to any lower order.

A co-ordinate frame $(X, Y)$ is considered, such that the frame is stationary relative to the frame of interest (often the sea floor), through which the waves propagate at velocity $c$. The origin is on the bed, with $X$ the horizontal co-ordinate in the direction of propagation of the waves, and with $Y$ vertical, such that $X=x+c t$, and $Y=y$. The fluid velocities in the $(X, Y)$ frame, $(U, V)$, are $U=\partial \Phi / \partial X, V=\partial \Phi / \partial Y$, in which the velocity potential, $\Phi$, can simply be obtained from Eq. 12:

$\Phi(X, Y, t)=(c-\bar{u}) X$

$+C_{o}\left(\frac{g}{k^{3}}\right)^{1 / 2} \sum_{i=1}^{5} \epsilon^{i} \sum_{j=1}^{i} A_{i j} \cosh j k Y \sin j k(X-c t) \ldots \ldots \ldots \ldots \ldots \ldots$

and, similarly, the expression for surface elevation from Eq. 14 is

$k \eta(X, t)=k d+\epsilon \cos k(X-c t)$

+ other terms in Eq. 14 with $x=X-c t \ldots \ldots \ldots \ldots \ldots \ldots$

In each of these expressions, the time origin is such that $t=0$ when a wave crest is at $X=0$. The pressure at any point, $p(X, Y, t)$, is given by $\frac{p(X, Y, t)}{\rho}=R-g Y-\frac{1}{2}\left[(U-c)^{2}+V^{2}\right] \ldots \ldots \ldots \ldots \ldots \ldots \ldots \ldots \ldots$

in which $\rho=$ the fluid density.

For the case of deep water, the expressions yield

$\Phi(X, Y, t)=(c-\bar{u}) X+\left(\frac{g}{k^{3}}\right)^{1 / 2}\left[\epsilon e^{k Y_{*}} \sin k(X-c t)\right.$

+ other terms in Eq. 17 with $x=X-c t]$

in which $\bar{u}$ is given by Eq. 19 ; and $Y_{*}=$ the elevation above the mean surface level. The elevation of the surface relative to the mean, $\eta_{*}(X, t)$, is given by

$k \eta_{*}(X, t)=\epsilon \cos k(X-c t)$

+ other terms in Eq. 18 with $x=X-c t$ 
For the pressure in deep water the equations yield

$\frac{p}{\rho}=\frac{g}{k}\left(\frac{1}{2}-k y *+\frac{1}{2} \epsilon^{2}+\frac{1}{4} \epsilon^{4}\right)-\frac{1}{2}\left[(U-c)^{2}+V^{2}\right]$

\section{Comparison With Experiment and with Accurate Solutions}

The most comprehensive set of results for the integral properties of periodic waves are those given by Cokelet (2), obtained from very highorder Stokes' expansions with extensive series-enhancement processing. These results provide a standard against which the accuracy of the present application-oriented low-order theory can be tested.

Of all integral quantities, that most often used as a basis for comparison is $\bar{u}$, which is the wave speed, $c$, for the special case $c_{E}=0$. Cokelet's results for this quantity are shown on Fig. 2, in which is plotted the square of the dimensionless wave speed, $c^{2} \mathrm{k} / \mathrm{g}$, against the dimensionless wave height, $k H / 2$, for several different values of the length scale, $k l=k Q / \bar{u}$, taken from Tables A0 and A2-A6 of Cokelet (2). The physical importance of $k l$ is not immediately obvious. However, it is approximately the dimensionless depth, so that the water depth is roughly constant for each curve. This gives the approximate wavelength/water depth ratios as shown on the figure to give a better idea of the actual wavelengths. Results from the present theory were found by setting the appropriate value of $k l$ from the values used by Cokelet, and then by solving an equation for $k$. This is similar to Eqs. 23 and 26 , but made up from the ratio $Q / \bar{u}$ into which Eqs. 13 and 15 for $\bar{u}$ and $Q$, respectively, have been substituted. From Fig. 2 it can be seen that for waves longer than about 10 times the water depth, the theory is not accurate, but for all waves shorter than this, the fifth-order theory agrees very closely with the accurate numerical results. This must be considered rather surprising in view of the fact that the latter had to be obtained from theories of very high order, with convergence-acceleration procedures. The phenomenon of the maxima in wave speed was not shown by the present results, but even for the highest waves, disagreement was not very large. For waves in deep water the accuracy is remarkable.

As the "wave speed' obtained previously is an integral quantity of the wave train (mean fluid velocity on a constant level), it might be expected that the details of actual flow fields would show somewhat poorer agreement. To examine this, comparisons were made with several of the experimental results for long waves of Le Mehaute, et al. (6), to which several other wave theories have been previously applied. Results are shown in Fig. 3, for the fluid velocity underneath the wave crests.

Rienecker and Fenton (8) have applied an accurate numerical method to these experimental results, using the appropriate condition $c_{S}=0$, and in their paper gave some examination to the experimental measurements and showed how they tend to be less than actual instantaneous flow velocities. Thus, rather more than the experimental results, the curves obtained by Rienecker and Fenton might be considered the real standard for comparison to see how accurately the present approximate theory solves the idealized two-dimensional incompressible irrotational problem. 


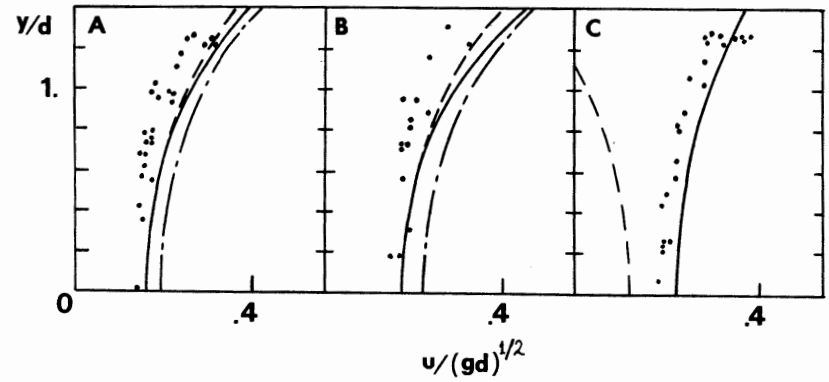

FIG. 3.-Horizontal Fluid Velocity Under Wave Crests, and Variation with Elevation Above Bed (Dots = Experimental Points of Lé Mehauté, et al.; Solid Line = Numerical Solution of Rienecker and Fenton; Irregular Dashed Line = Fifth-Order Solution of Skjelbreia and Hendrickson with $c_{E}=0$; Dashed Line $=$ Fifth-Order Solution from Present Theory; $A-\tau(g / d)^{1 / 2}=8.59, H / d=0.434 ; B-\tau(g / d)^{1 / 2}$ $\left.=8.59, H / d=0.499 ; C-\tau(g / d)^{1 / 2}=15.87, H / d=0.420\right)$

The first two cases show a dimensionless period of $\tau(g / d)^{1 / 2}=8.59$ (roughly equal to wavelength/depth), and wave heights of 0.434 and 0.499 times the water depth. It can be seen that the fifth-order Stokes theory agrees closely with the numerical results, gradually diverging for higher elevations. The fact that the present low-order theory is closer to the experimental results does not mean that it is a more accurate solution of the steady irrotational flow problem as set out in this paper. Rather the errors of the present theory tend to agree with errors caused by the manner in which the experiments were conducted. Also plotted in the figure are the curves, plotted by Le Mehaute et al., from the theory of Skjelbreia and Hendrickson, but where the inappropriate criterion of zero current $c_{E}=0$ was used. It can be seen that the results so obtained are in error by some $20-40 \%$, and that it is important to use the correct value of the wave speed.

Any confidence that the results instill, based on the first two cases, is quickly destroyed by those for the third, for the long wave of $\tau(g / d)^{1 / 2}$ $=15.87(\approx \lambda / d)$ and height $H / d=0.420$. In this case the expansion parameter, $\epsilon$, is only 0.08 , but the effective expansion parameter $\epsilon /(k d)^{3}$ is roughly 1.2, and the Stokes theory would not be expected to be accurate. Indeed, it is obviously grossly in error, and the result provides a clear warning that Stokes theories should not be used for long waves. A reasonable upper limit is $\tau(g / d)^{1 / 2} \approx \lambda / d \approx 10$. Beyond this limit, cnoidal wave theory can be used, for which reference can be made to Fenton (5) for a higher order theory (which uses $c_{E}=0$ ), and which was found to be satisfactory for long waves, but not for waves higher than half the depth. For high accuracy, over all wave heights and lengths short of the solitary wave, the numerical method given in Ref. 8 seems the most capable.

\section{Conclusions}

1. A fifth-order Stokes theory for periodic waves has been obtained which uses the actual wave steepness as expansion parameter. In ap- 
plications where the wavelength is initially unknown, one nonlinear equation must be solved as the first step, whereas previous theories have required the solution of two or three simultaneous equations, which has been found to be difficult.

2. It has been shown that to apply this theory or any other steady wave theory, more information is necessary than has usually been provided in the past. Neither this theory, nor any other, can give the actual wave speed in most situations, which is governed by factors external to the theory, such as the prevailing current or mass flux. If the wavelength is not known, either the wave speed or the mean current at a point in the fluid or the mass flux induced by the waves must be known, so that the wavelength can be obtained. If none of these are known, then application of the theory, or any other, is irrational and likely to be in error at first order, as in almost all previous applications of steady wave theories. In this case there is clearly no reason to use any theoretical results higher than first order.

3. A method has been devised for the numerical checking of perturbation expansions in engineering and physics. Using this it has been shown numerically that the present theory and that of Chappelear are correct, but that the theories of De and Skjelbreia and Hendrickson contain errors at fifth-order.

4. It has been shown that in the long wave limit, the expansion parameter for Stokes-type theories becomes the Ursell number, which is large in this limit so that the theories are inaccurate, which was first pointed out by Ursell (13). The present fifth-order theory has been shown to be quite accurate for waves shorter than 10 times the water depth. If high accuracy is required, or longer or higher waves are to be studied, the numerical method of Rienecker and Fenton (8) is preferable.

\section{Appendix I.-References}

1. Chappelear, J. E., "Direct Numerical Calculation of Wave Properties," Journal of Geophysical Research, Vol. 66, 1961, pp. 501-508.

2. Cokelet, E. D., "Steep Gravity Waves in Water of Arbitrary Uniform Depth," Philosophical Transcripts Royal Society of London, Series A, Vol. 286, 1977, pp. 183-230.

3. Dailey, J. E., "Stokes V Wave Computations in Deep Water," Journal of the Waterway, Port, Coastal and Ocean Division, ASCE, Vol. 104, No. WW4, Nov., 1978, pp. 447-453.

4. De, S. C., "Contributions to the Theory of Stokes Waves," Proceedings, Cambridge Philosophical Society, Vol. 51, 1955, pp. 713-736.

5. Fenton, J. D., "A High-Order Cnoidal Wave Theory," Journal of Fluid Mechanics, Vol. 94, 1979, pp. 129-161.

6. Le Mehaute, B., Divoky, D., and Lin, A., "Shallow Water Waves: A Comparison of Theories and Experiments," Proceedings, 11th Conference Coastal of Engineering, Vol. 1, 1968, pp. 86-107.

7. Nishimura, H., Isobe, M., and Horikawa, K., "Higher Order Solutions of the Stokes and Cnoidal Waves," Journal of the Faculty of Engineering, Univ. Tokyo, Series B, Vol. 34, 1977, pp. 267-293.

8. Rienecker, M. M., and Fenton, J. D., "A Fourier Approximation Method for Steady Water Waves," Journal of Fluid Mechanics, Vol. 104, 1981, pp. 119-137.

9. Schwartz, L. W., "Computer Extension and Analytic Continuation of Stokes' Expansion for Gravity Waves," Journal of Fluid Mechanics, Vol. 62, 1974, pp. 553-578. 
10. Schwartz, L. W., and Fenton, J. D., "Strongly-nonlinear Waves," Ann. Rev. Fluid Mech., Vol. 14, 1982, pp. 39-60.

11. Skjelbreia, L., and Hendrickson, J., "Fifth Order Gravity Wave Theory," Proceedings 7th Conference of Coastal Engineering, pp. 184-196.

12. Tsuchiya, Y., and Yamaguchi, M., "Some Considerations on Water Particle Velocities of Finite Amplitude Wave Theories," Coastal Engineering in Japan, Vol. 15, 1972, pp. 43-57.

13. Ursell, F., "The Long-Wave Paradox in the Theory of Gravity Waves," Proceedings, Cambridge Philosophical Society, Vol. 49, 1953, pp. 685-694.

\section{Appendix II.-Notation}

The following symbols are used in this paper:

$A_{i j}=$ dimensionless coefficients in series for $\Phi$ and $\phi$;

$a=$ length scale in leading term in Fourier series as used by Stokes;

$B_{i j}=$ dimensionless coefficients in Fourier series for $\eta(x)$;

$b_{i j}=$ dimensionless coefficients in power series for $\eta(x)$;

$C_{i}=$ dimensionless coefficients in series for $\bar{u}$;

$c=$ wave speed;

$c_{E}=$ mean current (mean Eulerian horizontal fluid velocity);

$c_{S}=$ mean fluid transport velocity;

$D_{i}=$ dimensionless coefficients in series for $Q$;

$d=$ mean depth of water;

$E_{i}=$ dimensionless coefficients in series for $R$;

$e_{j}=j$ th Fourier component of errors at free surface;

$f_{i j}=$ dimensionless coefficients in series for $\psi$;

$g=$ gravitational acceleration;

$H=$ wave height (crest to trough);

$k=$ wavenumber $=2 \pi / \lambda$;

$l=$ depth scale $=Q / \bar{u} ;$

$n(j)=$ exponent of $\epsilon$ in leading error term in boundary conditions;

$p=$ pressure;

$Q=$ volume flow rate of fluid relative to waves;

$R=$ Bernoulli constant in steady flow;

$S=\operatorname{sech} 2 k d$;

$U=$ horizontal component of fluid velocity, in particular frame of interest;

$u=$ ditto, in frame in which waves are stationary;

$\bar{u}=$ mean fluid speed (in frame in which waves are stationary) over one wavelength for constant elevation. This is equal to wave speed relative to frame in which there is zero current;

$V=$ vertical component of fluid velocity, in particular frame of interest;

$v=$ ditto, in frame in which waves are stationary;

$(X, Y)=$ Cartesian coordinates in frame of interest, through which waves move at speed $c ; X=x+c t, Y=y$;

$(x, y)=$ Cartesian coordinates in frame where waves are stationary;

$Y_{*}=y_{*}=$ elevation above mean fluid level;

$0=$ Landau order symbol, used as in $0\left(\epsilon^{6}\right)$, means neglected terms of errors are proportional to sixth power of $\epsilon$; 
$\alpha=$ coefficient of leading error term in boundary conditions;

$\boldsymbol{\epsilon}=k H / 2$, wave steepness;

$\eta=$ elevation of free surface above bottom;

$\eta_{*}=$ elevation of free surface above mean level;

$\lambda=$ wavelength;

$\rho=$ fluid density;

$\tau=$ wave period;

$\Phi=$ velocity potential in $(X, Y)$ frame;

$\phi=$ velocity potential in $(x, y)$ frame; and

$\psi=$ stream function in $(x, y)$ frame. 